\title{
Matrix-Valued and Quaternion Wavelets
}

P. Ginzberg and A. T. Walden, Senior Member, IEEE

Copyright (c) 2012 IEEE. Personal use of this material is permitted. However, permission to use this material for any other purposes must be obtained from the IEEE by sending a request to pubs-permissions@ieee.org. Paul Ginzberg and Andrew Walden are both at the Department of Mathematics, Imperial College London, London SW7 2BZ, UK. (e-mail: paul.ginzberg05@imperial.ac.uk and a.walden@imperial.ac.uk) 


\begin{abstract}
Wavelet transforms using matrix-valued wavelets (MVWs) can process the components of vectorvalued signals jointly. We construct some novel families of non-trivial orthogonal $n \times n$ MVWs for $n=2$ and 4 having several vanishing moments. Some useful uniqueness and non-existence results for filters with certain lengths and numbers of vanishing moments are proved. The matrix-based method for $n=4$ is used for the construction of a non-trivial symmetric quaternion wavelet with compact support. This is an important addition to the literature where existing quaternion wavelet designs suffer from some critical problems.
\end{abstract}

\title{
Keywords
}

multichannel wavelet, vector-valued wavelet, matrix-valued wavelet, multiwavelet, quaternion wavelet

\section{INTRODUCTION}

Multiresolution analysis (MRA) has been a highly successful field of research in recent decades. Wavelets [15] and multiwavelets [21] have found many applications in signal and image processing. In multichannel (vector-valued) signals, such as colour images and stereo sound, the channels are typically correlated, so that analyzing each channel separately with scalar wavelets is inappropriate.

Since multiwavelet transforms include a vectorization step, they can be applied directly to vector-valued signals. However, this generally leads to very poor results [16]. Wavelets and multiwavelets usually operate on scalar-valued functions depending on a variable such as time. $n \times n$ matrix-valued wavelets (MVWs) [30], [32], [35] have been applied to time-dependent $n$ vector-valued functions or $n \times n$ matrix-valued functions, respectively. As a result a plethora of other names have arisen, e.g., (multiple) vector-valued wavelet [8], [33], multichannel wavelet [1], [4], and wavelet with a full rank filter [2]. MVWs were introduced in [33], and generalisations have since been made, such as biorthogonal MVWs [2], [3], [8], [12], $m$-band MVWs [13], [9] and MVW packets [9].

Applications of MVWs include compression and denoising of colour images [1], [2] and of 2-D vector wind fields [20], [31]

With the exception of the biorthogonal filters given in [19, pp. 3-7] and [3, Table 4.1] (see also [2, p. 4]), explicit constructions for compactly-supported MVWs in the literature are very limited. Often the desire to obtain wavelet coefficients from the scaling coefficients in a simple 
closed form narrows design possibilities. This is the case in [8], [9], where only filters of length 3 are considered, in [12], [30] where ad-hoc constructions centered on the eigenvalues of the scaling filter transfer function are used, and in [13] where a symmetry condition is imposed. More general methods of designing MVWs include multichannel lifting schemes [2], [3], the spectral factorisation of interpolatory vector subdivision schemes [11], and directly solving the system of equations imposed by the design constraints [16], [19].

In this paper, we give for the first time, (as far as we are aware), families of non-trivial orthogonal MVWs for $n=2$ and 4 having several vanishing moments.

We focus on finite-length and orthogonal matrix-valued scaling filters (MVSFs). The content and structure of this paper is as follows. In Section II we distinguish carefully between matrix and vector multiresolution analyses and draw some conclusions about their applicability. Design equations for the MVW transform are discussed in Section III along with the important concept of orthogonal similarity for scaling filters, which adds constraints to the design equations. (The use of the set of design equations, enables us to prove several useful uniqueness and non-existence results for filters with certain lengths and numbers of vanishing moments.) Attention is paid in Section IV to the notion of trivial MVSFs, i.e., those that are orthogonally similar to a filter with block diagonal coefficients, and hence do not truly process all components jointly. Also considered is the computational burden for the corresponding MVW transform. Some useful non-existence results for short non-trivial filters are provided in Section V. For example, it is shown that every MVSF of length $L=4$ with two vanishing moments $(A=2)$ is trivial. In Section VI we construct a family of novel non-trivial MVSFs of length $L=6$ with $A=3$ for $n=2$. Section VII briefly considers how to derive wavelet filter coefficients from a decomposition of the polyphase matrix, and explicit wavelet filter coefficients are found corresponding to the $n=2$ scaling filter.

Since quaternions can be represented as structured matrices in $\mathbb{R}^{4 \times 4}$ under an isomorphism, we can treat quaternion wavelets as a special case of matrix-valued wavelets. We show in Section VIII that quaternion wavelets in one paper are invalid, the theory in another contains serious errors, while in Section IX we show that a third contains only trivial filters. We find non-trivial quaternion scaling and wavelet filters for $L=10$ and $A=5$ and plot the corresponding scaling and wavelet functions in Section X. Some closing comments are given in Section XIII. 


\section{Matrix And Vector Multiresolution Analyses}

\section{A. Basics}

$L^{2}\left(\mathbb{R}, \mathbb{R}^{n \times m}\right)$ is the space of $n \times m$ matrix-valued functions defined on $\mathbb{R}$ with values in $\mathbb{R}^{n \times m}$ having finite Frobenius norm. $n \times n$ matrix-valued wavelets (MVWs) have been used to construct multiresolution analysis (MRAs) of both $L^{2}\left(\mathbb{R}, \mathbb{R}^{n}\right)$ and $L^{2}\left(\mathbb{R}, \mathbb{R}^{n \times n}\right)$. We will clarify the relationship between these two approaches.

In the following, we will think of matrices as vectors of row vectors, i.e., $\mathbb{R}^{n \times n}=\left(\mathbb{R}^{1 \times n}\right)^{n}$ and $L^{2}\left(\mathbb{R}, \mathbb{R}^{n \times m}\right)=\left(L^{2}\left(\mathbb{R}, \mathbb{R}^{1 \times m}\right)\right)^{n}$.

Definition 1: A set $V$ is $\mathbb{R}^{n \times n}$-linear if it satisfies $\boldsymbol{x}, \boldsymbol{y} \in V \Rightarrow \boldsymbol{A x}+\boldsymbol{B} \boldsymbol{y} \in V \forall \boldsymbol{A}, \boldsymbol{B} \in \mathbb{R}^{n \times n}$. Of course $\mathbb{R}^{n \times n}$-linearity is stronger than $\mathbb{R}$-linearity.

Proposition 1: Every $\mathbb{R}^{n \times n}$-linear set of matrix-valued functions $V$ is of the form $V=S^{n}$, where $S$ is a linear set of (row-)vector-valued functions. Conversely, every set of this form is $\mathbb{R}^{n \times n}$-linear.

Proof: See Appendix-A.

We define the symbol inner product on $L^{2}\left(\mathbb{R}, \mathbb{R}^{n \times m}\right)$ by

$$
\left\langle\boldsymbol{F}_{1}, \boldsymbol{F}_{2}\right\rangle_{n \times n}=\int_{-\infty}^{\infty} \boldsymbol{F}_{1}(t) \boldsymbol{F}_{2}^{T}(t) \mathrm{d} t
$$

When $n>1$ this is not an inner product in the usual sense since it is $n \times n$-matrix-valued. However, it is bilinear, satisfies $\left\langle\boldsymbol{F}_{1}, \boldsymbol{F}_{2}\right\rangle_{n \times n}=\left\langle\boldsymbol{F}_{2}, \boldsymbol{F}_{1}\right\rangle_{n \times n}^{T}$, and the square of the Frobenius norm is $\|\boldsymbol{F}\|^{2}=\operatorname{tr}\left\{\langle\boldsymbol{F}, \boldsymbol{F}\rangle_{n \times n}\right\}$, where $\operatorname{tr}\{\cdot\}$ denotes trace.

In what follows $\boldsymbol{I}_{n}$ is the $n \times n$ identity matrix. $\mathbf{0}_{n \times n}$ is an $n \times n$ matrix of zeros and $\delta_{i, j}$ is the Kronecker delta.

Definition 2: An $(n \times n)$ matrix multiresolution analysis (MMRA) is a sequence of closed $\mathbb{R}^{n \times n}$-linear spaces $V_{j} \subset L^{2}\left(\mathbb{R}, \mathbb{R}^{n \times n}\right)$ satisfying

1. $V_{j} \subset V_{j-1} \forall j \in \mathbb{Z}$.

2. $\bigcup_{j \in \mathbb{Z}} V_{j}$ is dense in $L^{2}\left(\mathbb{R}, \mathbb{R}^{n \times n}\right)$ and $\bigcap_{j \in \mathbb{Z}} V_{j}=\left\{\mathbf{0}_{n \times n}\right\}$.

3. $\boldsymbol{F}(t) \in V_{0} \Leftrightarrow \boldsymbol{F}(t-k) \in V_{0} \forall k \in \mathbb{Z}$.

4. $\boldsymbol{F}(t) \in V_{j} \Leftrightarrow \boldsymbol{F}\left(2^{j} t\right) \in V_{0} \forall j \in \mathbb{Z}$.

5. There exists $\boldsymbol{\Phi} \in V_{0}$ such that it's integer translates form an orthonormal basis for $V_{0}$, in the sense that $\langle\boldsymbol{\Phi}(t-k), \boldsymbol{\Phi}(t-l)\rangle_{n \times n}=\delta_{k, l} \boldsymbol{I}_{n} \forall k, l \in \mathbb{Z}$ and for every $\boldsymbol{F} \in V_{0}$ there exists a sequence $\boldsymbol{A}_{k} \in \mathbb{R}^{n \times n}$ such that $\boldsymbol{F}(t)=\sum_{k \in \mathbb{Z}} \boldsymbol{A}_{k} \boldsymbol{\Phi}(t-k)$. 
$\boldsymbol{\Phi}$ is a (matrix) scaling function, and we say that $\mathbf{\Phi}$ generates the MMRA.

Definition 3: A vector multiresolution analysis (VMRA) is a sequence of closed linear spaces $V_{j} \subset L^{2}\left(\mathbb{R}, \mathbb{R}^{1 \times n}\right)$ satisfying

1. $V_{j} \subset V_{j-1} \forall j \in \mathbb{Z}$.

2. $\bigcup_{j \in \mathbb{Z}} V_{j}$ is dense in $L^{2}\left(\mathbb{R}, \mathbb{R}^{1 \times n}\right)$ and $\bigcap_{j \in \mathbb{Z}} V_{j}=\left\{\mathbf{0}_{1 \times n}\right\}$.

3. $\boldsymbol{f}(t) \in V_{0} \Leftrightarrow \boldsymbol{f}(t-k) \in V_{0} \forall k \in \mathbb{Z}$.

4. $\boldsymbol{f}(t) \in V_{j} \Leftrightarrow \boldsymbol{f}\left(2^{j} t\right) \in V_{0} \forall j \in \mathbb{Z}$.

5. There exist $\phi_{1}, \ldots, \phi_{n} \in V_{0}$ such that their integer translates form an orthonormal basis for $V_{0}$.

The $\phi_{i}$ are vector scaling functions, and we say that they generate the VMRA.

Proposition 2: An $n \times n$ matrix-valued function $\mathbf{\Phi}$ generates a MMRA $\left\{V_{j}\right\}$ if and only if its rows generate a VMRA $\left\{S_{j}\right\}$. Furthermore, we then have $V_{j}=S_{j}^{n}$.

Proof: The proof is given in Appendix-A.

\section{B. Interpretation and Usage}

MMRAs are convenient, since they allow a theory and notation that parallels scalar wavelets. However, we cannot say that matrix-valued wavelets are particularly well suited for analysing matrix-valued signals. From Definition 2, point 5, we see that

$$
\boldsymbol{A}_{k}=\langle\boldsymbol{F}, \boldsymbol{\Phi}(t-k)\rangle=\int_{-\infty}^{\infty} \boldsymbol{F}(t) \boldsymbol{\Phi}^{T}(t-k) \mathrm{d} t
$$

i.e., the MVW transform only requires multiplication by matrices from the right, thus operating on each row of the signal independently as a vector (row $i$ of $\boldsymbol{A}_{k}$ only involves row $i$ of $\boldsymbol{F}$ ). So the matrix-valued wavelet transform is equivalent to $n$ independent vector-valued wavelet transforms. If we wish to jointly transform the rows of an $n \times n$ matrix-valued signal then we should treat it as a $n^{2}$ dimensional vector-valued signal, and use an $n^{2} \times n^{2} \mathrm{MVW}$.

One application where the MMRA formulation is particularly useful is in the design of hypercomplex wavelets. Complex numbers, quaternions and other associative hypercomplex algebras are isomorphic to subalgebras of $\mathbb{R}^{n \times n}$. This allows us to treat hypercomplex wavelets as a special case of matrix-valued wavelets. We will use this in Section VIII to design quaternion wavelets. 


\section{Design EQUATIONS FOR THE MVW TRANSFORM}

\section{A. Basics}

Since $\boldsymbol{\Phi}(t) \in V_{0} \subset V_{-1}$, it satisfies the two-scale dilation equation

$$
\boldsymbol{\Phi}(t)=\sqrt{2} \sum_{k \in \mathbb{Z}} \boldsymbol{G}_{k} \boldsymbol{\Phi}(2 t-k) .
$$

With $\hat{x}$ denoting the Fourier transform of $x$, we have, in the frequency domain,

$$
\hat{\mathbf{\Phi}}(f)=\frac{1}{\sqrt{2}} \hat{\boldsymbol{G}}\left(\frac{f}{2}\right) \hat{\mathbf{\Phi}}\left(\frac{f}{2}\right)
$$

where,

$$
\hat{\boldsymbol{\Phi}}(f)=\int_{-\infty}^{\infty} \boldsymbol{\Phi}(t) \mathrm{e}^{-\mathrm{i} 2 \pi f t} \mathrm{~d} t ; \quad \hat{\boldsymbol{G}}(f)=\sum_{k \in \mathbb{Z}} \boldsymbol{G}_{k} \mathrm{e}^{-\mathrm{i} 2 \pi f k} .
$$

$\left\{\boldsymbol{G}_{k}, k \in \mathbb{Z}\right\}$ is the matrix-valued scaling filter (MVSF). We are interested in compactlysupported wavelets where the sequence is $\ldots, \mathbf{0}_{n \times n}, \boldsymbol{G}_{0}, \ldots, \boldsymbol{G}_{L-1}, \mathbf{0}_{n \times n}, \ldots$ Here $L$ is the finite length of the filter and each $\boldsymbol{G}_{k}=\left(g_{i, j, k}\right)_{i, j=1 \ldots n}$ is an $n \times n$ matrix.

With $\left\{\boldsymbol{G}_{k}\right\}$ of finite length, we know $\boldsymbol{\Phi}(t)$ has compact support [27, p. 185] and hence $\hat{\mathbf{\Phi}}(f)$ is continuous. Iterating $(2)$ and with $\hat{\boldsymbol{\Phi}}(f)$ continuous at $f=0$, and equal to $\boldsymbol{I}_{n}$,

$$
\hat{\boldsymbol{\Phi}}(f)=\prod_{m=1}^{\infty} \frac{\hat{\boldsymbol{G}}\left(f / 2^{m}\right)}{\sqrt{2}} .
$$

The order of the product in (4), which expands from left to right, is important. Result (4) allows us to compute the scaling function from the filter coefficients and we may concentrate on designing the filter $\left\{\boldsymbol{G}_{k}\right\}$. We will express the design conditions we wish to impose on the scaling and wavelet functions directly in terms of equations in the scaling filter coefficients $\boldsymbol{G}_{k}$.

\section{B. Necessary Constraints}

Since filter lengths may be odd or even, for our design equations we define $L^{\prime}$ always to be even. For a filter with even length $L$ we set $L^{\prime}=L$ and for a filter with odd length $L$ we set $L^{\prime}=L+1$ with the constraint $\boldsymbol{G}_{L}=\mathbf{0}_{n \times n}$.

Setting $f=0$ in (2) we deduce that

$$
\hat{\boldsymbol{G}}(0)=\sum_{k=0}^{L^{\prime}-1} \boldsymbol{G}_{k}=\sqrt{2} \boldsymbol{I}_{n} .
$$

The condition (5) arises naturally for MVWs, and in the context of such transforms [30] noted that (5) implies that the filter $\left\{\boldsymbol{G}_{k}\right\}$ preserves constant signals. The condition sets MVWs apart 
from standard multiwavelets, where one eigenvalue of $\hat{\boldsymbol{G}}(0) / \sqrt{2}$ is equal to 1 , and it is usually assumed that all other eigenvalues are strictly less than 1 in absolute value.

Proposition 3: If $\left\{\boldsymbol{G}_{k}\right\}$ is a finite-length filter satisfying (5), then the infinite product (4) converges uniformly on compact sets.

Proof: This follows from [18, Proposition 5.2], since from (5), $\left(\frac{\hat{\boldsymbol{G}}(0)}{\sqrt{2}}\right)^{\infty}=\boldsymbol{I}_{n}$. We hence see that $\hat{\boldsymbol{\Phi}}(f)$ in (4) is well-defined.

Necessary, but not sufficient, conditions for orthonormality of the scaling function are

$$
\sum_{k=0}^{L^{\prime}-1-2 m} \boldsymbol{G}_{k} \boldsymbol{G}_{k+2 m}^{T}=\delta_{m, 0} \boldsymbol{I}_{n}, m=0, \ldots,\left(L^{\prime} / 2\right)-1 .
$$

A sufficient condition is however given by

Proposition 4: Let $\left\{\boldsymbol{G}_{k}\right\}$ be a finite length filter satisfying (5) and (6). If $\operatorname{det}(\hat{\boldsymbol{G}}(f)) \neq 0$ for $|f| \leq \frac{1}{4}$, then $\boldsymbol{\Phi}(t)$ is a matrix-valued scaling function for a MMRA.

Proof: This is a reformulation of [32, Theorem 3.4] which requires that $\inf _{|f|<\frac{1}{4}}|\lambda(f)|>0$ for all eigenvalue functions $\lambda(f)$ of $\hat{\boldsymbol{G}}(f)$. However, all eigenvalues are non-zero iff the determinant is non-zero. Also, the eigenvalues are bounded and the determinant is a continuous function of $f$.

Unlike [30], we will not include this technical sufficient condition in the design. However, in this form it is simple to calculate and check for a given scaling filter, and we found that all constructions in this paper satisfy it.

In the following we deal exclusively with finite-length filters satisfying the necessary constraints (5) and (6), i.e., our MVSFs are assumed orthogonal.

The MVSF has $A$ vanishing moments if [27, Section 7.1]

$$
\sum_{k=0}^{L^{\prime}-1}(-1)^{k} k^{d} \boldsymbol{G}_{k}=\mathbf{0}_{n \times n}, d=0, \ldots, A-1 .
$$

Those of [30] had only one vanishing moment.

Remark 1: As in the scalar case, the vanishing moment condition (7) is satisfied iff $\hat{\boldsymbol{G}}$ admits the factorization $\hat{\boldsymbol{G}}(f)=\left(1+\mathrm{e}^{-\mathrm{i} 2 \pi f}\right)^{A} \hat{\boldsymbol{J}}(f)$ for some filter $\left\{\boldsymbol{J}_{k}\right\}$ of length $L-A$. It is also equivalent to polynomial reproduction of order $A$ for the scaling filter [3, Theorem 3.1].

Proposition 5: Every MVSF has at least one vanishing moment.

Proof: The proof is given in [30, eqn. (2.5)]. 


\section{Orthogonal Similarity}

Definition 4: Two filters $\left\{\boldsymbol{G}_{k}\right\}$ and $\left\{\boldsymbol{J}_{k}\right\}$ are orthogonally similar iff

$$
\boldsymbol{G}_{k}=\boldsymbol{O} \boldsymbol{J}_{k} \boldsymbol{O}^{T}, \forall k \in \mathbb{Z}
$$

for some orthogonal matrix $\boldsymbol{O}$, (i.e., $\boldsymbol{O} \boldsymbol{O}^{T}=\boldsymbol{I}_{n}$ ).

The map $\left\{\boldsymbol{J}_{k}\right\} \mapsto\left\{\boldsymbol{O J}_{k} \boldsymbol{O}^{T}\right\}$ is called an orthogonal similarity transformation (OST).

Proposition 6: If $\left\{\boldsymbol{G}_{k}\right\}$ is an MVSF of length $L$ with $A$ vanishing moments, then any orthogonally similar filter $\left\{\boldsymbol{O} \boldsymbol{G}_{k} \boldsymbol{O}^{T}\right\}$ is also an MVSF of length $L$ with $A$ vanishing moments. If furthermore the multivariate scaling function $\boldsymbol{\Phi}$ associated with $\left\{\boldsymbol{G}_{k}\right\}$ generates a MMRA $\left\{V_{j}\right\}$, then the multivariate scaling function associated with $\left\{\boldsymbol{O} \boldsymbol{G}_{k} \boldsymbol{O}^{T}\right\}$ generates the MMRA $\left\{V_{j} \boldsymbol{O}^{T}\right\}$, where $V_{j} \boldsymbol{O}^{T}=\left\{\boldsymbol{F} \boldsymbol{O}^{T}: \boldsymbol{F} \in V_{j}\right\}$.

Proof: The proof is given in Appendix-A.

Lemma 1: Every $\boldsymbol{M} \in \mathbb{R}^{n \times n}$ is orthogonally similar to a matrix of the form $\boldsymbol{D}+\boldsymbol{A}$, where $\boldsymbol{D}$ is diagonal and $\boldsymbol{A}$ is anti-symmetric, (i.e., $\boldsymbol{A}=-\boldsymbol{A}^{T}$ ).

Proof: $\quad \boldsymbol{M}=\boldsymbol{S}+\boldsymbol{B}$ where $\boldsymbol{S}=\frac{1}{2}\left(\boldsymbol{M}+\boldsymbol{M}^{T}\right)$ is symmetric and $\boldsymbol{B}=\frac{1}{2}\left(\boldsymbol{M}-\boldsymbol{M}^{T}\right)$ is anti-symmetric. By the eigenvalue decomposition, $\boldsymbol{S}=\boldsymbol{O} \boldsymbol{D} \boldsymbol{O}^{T}$ for some orthogonal matrix $\boldsymbol{O}$ and diagonal matrix $\boldsymbol{D} . \boldsymbol{M}$ is orthogonally similar to $\boldsymbol{O}^{T} \boldsymbol{M} \boldsymbol{O}=\boldsymbol{D}+\boldsymbol{A}$ where $\boldsymbol{A}=\boldsymbol{O}^{T} \boldsymbol{B} \boldsymbol{O}=$ $-\boldsymbol{O}^{T} \boldsymbol{B}^{T} \boldsymbol{O}=-\boldsymbol{A}^{T}$ is anti-symmetric.

Corollary 1: Since we can obtain from any given filter a family of orthogonally similar filters, when designing filters we can, without loss of generality, assume that $\boldsymbol{G}_{0}$ is of the form $\boldsymbol{G}_{0}=$ $\boldsymbol{D}+\boldsymbol{A}$ with $\boldsymbol{D}$ diagonal and $\boldsymbol{A}$ anti-symmetric, i.e., we add the design equations

$$
g_{i, j, 0}=-g_{j, i, 0} \quad \text { for } \quad i \neq j .
$$

Remark 2: There will be possibly more than one filter in the orthogonally-similar family of filters satisfying (9).

\section{Construction of MVSFs}

\section{A. Trivial Matrix-valued Scaling Filters}

Suppose we have $n$ scalar-valued scaling filters $\left\{c_{1, k}\right\}, \ldots,\left\{c_{n, k}\right\}$ of length $L$ (or less). We may construct a MVSF $\left\{\boldsymbol{G}_{k}\right\}$ with diagonal coefficients, by setting $g_{i, i, k}=c_{i, k}$ for each $i=1, \ldots, n$. Conversely, any MVSF with diagonal coefficients is of this form. Applying a diagonal filter to a 
vector-valued signal is equivalent to using independent scalar filters on each of the channels of the vector-valued signal.

More generally, given an $n_{1} \times n_{1}$ scaling filter $\left\{\boldsymbol{G}_{k}\right\}$ of length $L_{1}$ with $A_{1}$ vanishing moments and a $n_{2} \times n_{2}$ scaling filter $\left\{\boldsymbol{J}_{k}\right\}$ of length $L_{2}$ with $A_{2}$ vanishing moments, we can construct a $\left(n_{1}+n_{2}\right) \times\left(n_{1}+n_{2}\right)$ scaling filter $\left\{\boldsymbol{G}_{k} \oplus \boldsymbol{J}_{k}\right\}$, where the direct sum of two matrices is

$$
\boldsymbol{G}_{k} \oplus \boldsymbol{J}_{k}=\left[\begin{array}{cc}
\boldsymbol{G}_{k} & \mathbf{0} \\
\mathbf{0} & \boldsymbol{J}_{k}
\end{array}\right],
$$

and the filter will have length $\max \left\{L_{1}, L_{2}\right\}$ and $\min \left\{A_{1}, A_{2}\right\}$ vanishing moments. Such a block diagonal filter applies $\left\{G_{k}\right\}$ to the first $n_{1}$ dimensions, and independently, $\left\{J_{k}\right\}$ to the latter $n_{2}$ dimensions.

Definition 5: A filter is trivial if it is orthogonally similar to a filter with block diagonal coefficients, i.e., to the direct sum of two or more filters.

If we wish to impose on the trivial filter properties which are invariant under OSTs, such as short support, vanishing moments and symmetry, then all filters from which it is constructed must have these properties.

Trivial filters may have desirable properties which are absent in the filters from which they are assembled. However, any such properties will be basis-dependent. For example, given any scalar-valued scaling filter $\left\{c_{k}\right\}$ of length $L$, the trivial filter given by $\boldsymbol{G}_{k}=\boldsymbol{O}\left(c_{k} \oplus c_{L-k-1}\right) \boldsymbol{O}^{T}$ is

$$
\boldsymbol{G}_{k}=\frac{1}{2}\left[\begin{array}{ll}
c_{k}+c_{L-k-1} & c_{k}-c_{L-k-1} \\
c_{k}-c_{L-k-1} & c_{k}+c_{L-k-1}
\end{array}\right],
$$

where

$$
\boldsymbol{O}=\frac{1}{\sqrt{2}}\left[\begin{array}{cc}
1 & 1 \\
1 & -1
\end{array}\right],
$$

and satisfies the symmetric-antisymmetric (SA) condition, [10], $\boldsymbol{G}_{k}=\boldsymbol{P}_{2} \boldsymbol{G}_{L-k-1} \boldsymbol{P}_{2}$, where $\boldsymbol{P}_{n}$ is the $n \times n$ diagonal matrix with $(i, i)$ entry $(-1)^{i+1}$. On the other hand, since symmetry is preserved by OSTs, symmetric filters cannot be obtained in this fashion from asymmetric filters.

Trivial filters require less computation than non-trivial filters. If for example the scaling and wavelet filters are orthogonally similar to diagonal filters, then we may implement the MVWT by first applying an orthogonal change of basis to the data, (using the $\boldsymbol{O}$ in (8)), then computing $n$ independent scalar wavelet transforms, and finally inverting the change of basis. As shown in Table I, this implementation of the trivial MVWT speeds up the algorithm by a factor of at 


\begin{tabular}{|l||l|l|}
\hline filter type & multiplications & additions \\
\hline diagonal & $n L N$ & $n(L-1) N$ \\
trivial & $n(L+2 n) N$ & $n(L+2 n-3) N$ \\
non-trivial & $n^{2} L N$ & $n(n L-1) N$ \\
\hline
\end{tabular}

TABLE I

NUMBER OF OPERATIONS REQUIRED FOR A ONE-LEVEL $n \times n$ MATRIX WAVELET TRANSFORM WHEN THE SCALING AND WAVELET FILTERS ARE DIAGONAL, DIAGONAL UP TO ORTHOGONAL SIMILARITY (trivial) or general (non-trivial). Here $L$ is the Length of the filters and $N$ is the LENGTH OF THE VECTOR-VALUED INPUT.

least $\frac{n L}{2 n+L}$ compared to a general MVWT. If multiple levels of the transform are required, then the improvement is greater, since the changes of basis need to be applied once only.

\section{B. Non-trivial Matrix-valued Scaling Filters}

Previous constructions of compactly-supported orthogonal MVWs (e.g. [30]) concentrate on satisfying the conditions of Proposition 4. As a result, the ad-hoc form of the scaling filters limits the achievable properties. Another method obtains scaling filters through the spectral factorization of interpolatory filters [11], however, this requires that we design interpolatory filters with good properties. For biorthogonal MVWs, constructions based on the lifting scheme are also available [2], [3].

As in [16], [19], we will design the scaling filters $\left\{\boldsymbol{G}_{k}\right\}$ by directly solving a set of design equations. One of the advantages of this approach is that we can use it to prove uniqueness and non-existence results.

We will first treat the cases $L=2,3$ and 4 by hand, since this allows us to prove non-existence results for all $n$, and rely on computational results for all latter cases.

\section{SOME NON-EXISTENCE RESUlts FOR SHORT NON-TRIVIAL MVSFs}

First, a general result:

Proposition 7: MVSFs which are symmetric or symmetric-antisymmetric are of even length.

Proof: The proof is given in Appendix-A. 
We now make some observations for short filter lengths. For $L=2$ and $A=1,(5)$ and (7) give us the trivial scaling filter built from the Haar filter, $\boldsymbol{G}_{0}=\boldsymbol{G}_{1}=\boldsymbol{I}_{n} / \sqrt{2}$, as the only solution.

\section{A. Length 3 with 1 Vanishing Moment}

Proposition 8: There are no non-trivial $n \times n$ MVSFs of length $L=3$ for $n \geq 2$.

Proof: By Proposition 5 there is a vanishing moment. Then (5) and (7) give us

$$
\begin{aligned}
\boldsymbol{G}_{0}+\boldsymbol{G}_{1}+\boldsymbol{G}_{2} & =\sqrt{2} \boldsymbol{I}_{n} \\
\boldsymbol{G}_{0}-\boldsymbol{G}_{1}+\boldsymbol{G}_{2} & =\mathbf{0}_{n \times n} .
\end{aligned}
$$

from which we deduce that

$$
\begin{aligned}
& \boldsymbol{G}_{1}=2^{-\frac{1}{2}} \boldsymbol{I}_{n} \\
& \boldsymbol{G}_{2}=2^{-\frac{1}{2}} \boldsymbol{I}_{n}-\boldsymbol{G}_{0} .
\end{aligned}
$$

Substituting these into (6) we get

$$
\begin{gathered}
2 \boldsymbol{G}_{0} \boldsymbol{G}_{0}^{T}-2^{-\frac{1}{2}}\left(\boldsymbol{G}_{0}+\boldsymbol{G}_{0}^{T}\right)=\mathbf{0}_{n \times n} \\
\boldsymbol{G}_{0} \boldsymbol{G}_{0}^{T}=2^{-\frac{1}{2}} \boldsymbol{G}_{0} .
\end{gathered}
$$

Substituting (14) into (13) we have $\boldsymbol{G}_{0}=\boldsymbol{G}_{0}^{T}$. By Corollary 1, up to an OST, $\boldsymbol{G}_{0}$ is thus diagonal. By (11) and (12), $\boldsymbol{G}_{1}$ and $\boldsymbol{G}_{2}$ are then also diagonal.

Corollary 2: Every $n \times n$ MVSF of length 3 has form $\boldsymbol{G}_{k}=\boldsymbol{O}\left(d_{k} \boldsymbol{I}_{m} \oplus d_{2-k} \boldsymbol{I}_{n-m}\right) \boldsymbol{O}^{T}$ where $\boldsymbol{O}$ is an orthogonal matrix, $0 \leq m \leq n$, and $\left\{d_{k}\right\}$ are the coefficients of the scalar Haar filter, i.e. $d_{0}=d_{1}=2^{-\frac{1}{2}}$ and $d_{k}=0$ otherwise.

Proof: This follows from Proposition 8, and the fact that the only scalar scaling filters with $L=3$ are the $L=2$ Haar filter followed by a zero and the $L=2$ Haar filter preceded by a zero. (Also note that the order of the diagonal elements is unimportant, since permutation matrices are orthogonal.)

In [8, Example 2], the following non-trivial orthogonal $2 \times 2$ scaling filter with $L=3$ is given:

$$
\begin{aligned}
& \boldsymbol{G}_{0}=\frac{1}{4 \sqrt{2}}\left[\begin{array}{ll}
2+\sqrt{5} & 0 \\
2-\sqrt{5} & 0
\end{array}\right], \boldsymbol{G}_{2}=\frac{1}{4 \sqrt{2}}\left[\begin{array}{ll}
0 & 2-\sqrt{5} \\
0 & 2+\sqrt{5}
\end{array}\right], \\
& \boldsymbol{G}_{1}=\frac{1}{4 \sqrt{2}}\left[\begin{array}{ll}
2+\sqrt{3} & 2-\sqrt{3} \\
2-\sqrt{3} & 2+\sqrt{3}
\end{array}\right]
\end{aligned}
$$


However, this filter has neither a vanishing moment nor satisfies (5). Proposition 5 is not contradicted: the filter is associated to a multiwavelet scaling function and a MRA of $L^{2}(\mathbb{R}, \mathbb{R})$, not a matrix-valued scaling function or MMRA of $L^{2}\left(\mathbb{R}, \mathbb{R}^{2 \times 2}\right)$.

\section{B. Length 4 with 2 Vanishing Moments}

Proposition 9: There are no non-trivial $n \times n$ MVSFs of length $L=4$ with $A=2$ vanishing moments for $n \geq 2$.

Proof: The linear system given by (5) and (7) simplifies to

$$
\boldsymbol{G}_{1}=2^{-3 / 2} \boldsymbol{I}_{n}+\boldsymbol{G}_{0} ; \boldsymbol{G}_{2}=2^{-1 / 2} \boldsymbol{I}_{n}-\boldsymbol{G}_{0} ; \boldsymbol{G}_{3}=2^{-3 / 2} \boldsymbol{I}_{n}-\boldsymbol{G}_{0}
$$

We can now write the orthogonality conditions (6) in terms of $\boldsymbol{G}_{0}$ :

$$
\begin{aligned}
4 \boldsymbol{G}_{0} \boldsymbol{G}_{0}^{T}-2^{-1 / 2}\left(\boldsymbol{G}_{0}+\boldsymbol{G}_{0}^{T}\right)+3 \cdot 2^{-2} \boldsymbol{I}_{n \times n} & =\boldsymbol{I}_{n \times n} \\
-2 \boldsymbol{G}_{0} \boldsymbol{G}_{0}^{T}+3 \cdot 2^{-3 / 2} \boldsymbol{G}_{0}-2^{-\frac{3}{2}} \boldsymbol{G}_{0}^{T}+2^{-3} \boldsymbol{I}_{n \times n} & =\mathbf{0}_{n \times n} .
\end{aligned}
$$

Adding $2^{-1 / 2}$ times the first equation to $2^{1 / 2}$ times the second, we deduce $\boldsymbol{G}_{0}=\boldsymbol{G}_{0}^{T}$ which by Corollary 1 means that, up to an OST, $\boldsymbol{G}_{0}$ is diagonal. By (15), $\boldsymbol{G}_{1}, \boldsymbol{G}_{2}$ and $\boldsymbol{G}_{3}$ are then also diagonal.

Corollary 3: Every $n \times n$ MVSF of length 4 with 2 vanishing moments has form $\boldsymbol{G}_{k}=$ $\boldsymbol{O}\left(d_{k} \boldsymbol{I}_{m} \oplus d_{3-k} \boldsymbol{I}_{n-m}\right) \boldsymbol{O}^{T}$ where $\boldsymbol{O}$ is an orthogonal matrix, $0 \leq m \leq n$, and $\left\{d_{k}\right\}$ is the scalar Daubechies extremal phase or minimum delay scaling filter of length 4 :

$$
d_{0}=\frac{1+\sqrt{3}}{4 \sqrt{2}} ; d_{1}=\frac{3+\sqrt{3}}{4 \sqrt{2}} ; d_{2}=\frac{3-\sqrt{3}}{4 \sqrt{2}} ; d_{3}=\frac{1-\sqrt{3}}{4 \sqrt{2}},
$$

and $d_{k}=0$ otherwise, [15].

Proof: This Daubechies filter and its time-reversed (maximum delay) version are the only scalar scaling filters of length 4 with 2 vanishing moments. Again, the order of the diagonal elements is unimportant.

\section{EXAMPLES OF NON-TRIVIAL MVSFs}

Consider $L$ even so that $L^{\prime}=L$. Each matrix equation is equivalent to a set of $n^{2}$ scalar equations. Hence the necessary conditions (5) and (6) give us $n^{2}[(L / 2)+1]$ scalar quadratic equations. The vanishing moment conditions (7) add $n^{2} A$ scalar linear equations, and the assumption $\boldsymbol{G}_{0}=\boldsymbol{D}+\boldsymbol{A}$ corresponds to $n(n-1) / 2$ scalar linear equations. Hence, the design equations describe a system of $n^{2}[A+(L+3) / 2]-(n / 2)$ quadratic equations in $n^{2} L$ variables. 
Having chosen a dimension $n$, length $L$, number of vanishing moments $A$ and any additional conditions that can be expressed as polynomial equations in the $g_{i, j, k}$, we can attempt to solve the resulting system of polynomial equations through symbolic computation. The approach used here is to pre-process the system of equations by computing a lexicographic Gröbner basis. This is a set of polynomials which has the same roots as our original system, but can be solved using a form of back-substitution. For some background on Gröbner bases, see [23], where a similar approach is used to design multiwavelets.

Two steps were included in the computation with Maple:

- The assumption $\boldsymbol{G}_{0}=\boldsymbol{D}+\boldsymbol{A}$ was included by making substitutions, reducing the number of variables, equivalent to setting $g_{i, j, 0}=-g_{j, i, 0}$ for all $i>j$.

- When computing the Gröbner basis, to avoid the presence of the irrational constant $\sqrt{2}$ in the system of equations, the coefficients $g_{i, j, k}$ were rescaled by a factor of $\sqrt{2}$, and adjusted after solution.

For $L=6, A=3$ and $n=2$ the non-trivial scaling filters are given (up to orthogonal similarity) by

$$
\begin{aligned}
& \boldsymbol{G}_{0}=\frac{1}{32 \sqrt{2}}\left[\begin{array}{cc}
x^{2}-2 x-3 & y \\
-y & x^{2}+2 x-3
\end{array}\right], \\
& \boldsymbol{G}_{1}=\frac{1}{32 \sqrt{2}}\left[\begin{array}{cc}
x^{2}-6 x+5 & y \\
-y & x^{2}+6 x+5
\end{array}\right], \\
& \boldsymbol{G}_{2}=\frac{1}{16 \sqrt{2}}\left[\begin{array}{cc}
-x^{2}-2 x+15 & -y \\
y & -x^{2}+2 x+15
\end{array}\right], \\
& \boldsymbol{G}_{3}=\frac{1}{16 \sqrt{2}}\left[\begin{array}{cc}
-x^{2}+2 x+15 & -y \\
y & -x^{2}-2 x+15
\end{array}\right], \\
& \boldsymbol{G}_{4}=\frac{1}{32 \sqrt{2}}\left[\begin{array}{cc}
x^{2}+6 x+5 & y \\
-y & x^{2}-6 x+5
\end{array}\right], \\
& \boldsymbol{G}_{5}=\frac{1}{32 \sqrt{2}}\left[\begin{array}{cc}
x^{2}+2 x-3 & y \\
-y & x^{2}-2 x-3
\end{array}\right],
\end{aligned}
$$

where $y=\sqrt{ }\left(-x^{4}+10 x^{2}+15\right)$, and $x \geq 0$ is a free parameter. Since $y$ must be real, the free parameter is limited to $x \leq \sqrt{ }(5+2 \sqrt{10}) \approx 3.3652$. The range of $y$ is $y \in[0,2 \sqrt{10}] \approx[0,6.3246]$, 

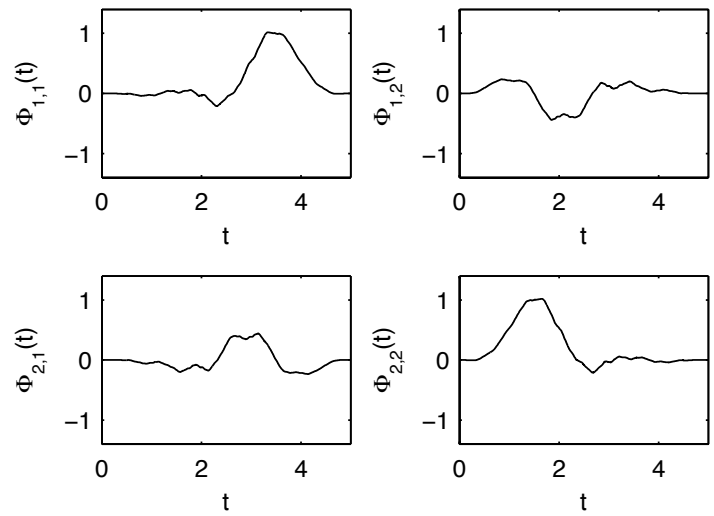

Fig. 1. Elements of $\boldsymbol{\Phi}(t)$ for the design with $L=6, A=3, n=2$ for the choice $x=\sqrt{5}$.

with the maximum reached at $x=\sqrt{5}$. The minimum, $y=0$, is reached for $x=\sqrt{ }(5+2 \sqrt{10})$, and gives the diagonal scaling filters built from the Daubechies extremal phase or minimum delay filter of length 6 [15, Table 6.1] and its time-reversed version. For all $x, \boldsymbol{G}_{k}=\boldsymbol{O}_{0} \boldsymbol{G}_{5-k} \boldsymbol{O}_{0}^{T}$ for $k=3,4,5$, with $\boldsymbol{O}_{0}=\left[\begin{array}{cc}0 & 1 \\ -1 & 0\end{array}\right]$.

For $x=\sqrt{5}, \mathbf{\Phi}(t)$ was computed from the $\boldsymbol{G}_{k}$ 's in (16) using the methodology in [30, Appendix $\mathrm{A}(\mathrm{a})]$, and is shown in Fig. 1.

Now the transformation

$$
a+b \mathrm{i} \mapsto\left[\begin{array}{cc}
a & -b \\
b & a
\end{array}\right]
$$

is an isomorphism between $\mathbb{C}$ and the subalgebra of complex-structured matrices in $\mathbb{R}^{2 \times 2}$. Choosing $x=0$ in the $\boldsymbol{G}_{k}$ 's gives us (up to a factor of $\sqrt{ } 2$ ) the real matrix representation (17) of the complex-valued Daubechies filter of length 6 which is symmetric [24, p. 222].

For $L=6, A=3$ and $n=3$ there are no non-trivial solutions.

\section{FROM SCALING FILTERS TO WAVELET FILTERS}

For a VMRA $\left\{V_{j}\right\}$, let $W_{j}$ be the orthogonal complement of $V_{j}$ in $V_{j-1}$. Then $\bigcup_{j \in \mathbb{Z}} W_{j}=$ $\bigcup_{j \in \mathbb{Z}} V_{j}$ and is dense in $L^{2}\left(\mathbb{R}, \mathbb{R}^{1 \times n}\right)$. We wish to find a matrix-valued wavelet $\Psi$ such that the integer translates of its rows form an orthonormal basis for $W_{0}$. Since $W_{0} \subset V_{-1}$, this is equivalent to finding a wavelet filter $\left\{\boldsymbol{H}_{k}\right\}$ such that $\boldsymbol{\Psi}(t)=\sqrt{2} \sum_{k \in \mathbb{Z}} \boldsymbol{H}_{k} \boldsymbol{\Phi}(2 t-k)$.

In the scalar case, $n=1$, it is well known that a wavelet filter $\left\{H_{k}\right\}$ can be obtained from its 
corresponding scaling filter $\left\{G_{k}\right\}$ by the quadrature mirror relationship

$$
H_{k}=(-1)^{k+1} G_{L-1-k}, \quad k=0, \ldots, L-1,
$$

but as discussed in [33], this only works for $n>1$ if $\hat{\boldsymbol{G}}(f)$ commutes with $\hat{\boldsymbol{G}}\left(f+\frac{1}{2}\right)$. In the general case, [33] suggests using paraunitary completion.

Consider the polyphase components of the scaling and wavelet filters (Fourier transforms of even and odd numbered terms)

$$
\begin{gathered}
\hat{\boldsymbol{G}}_{0}(f)=\sum_{k \in \mathbb{Z}} \boldsymbol{G}_{2 k} \mathrm{e}^{-\mathrm{i} 2 \pi f k}, \hat{\boldsymbol{G}}_{1}(f)=\sum_{k \in \mathbb{Z}} \boldsymbol{G}_{2 k+1} \mathrm{e}^{-\mathrm{i} 2 \pi f k} \\
\hat{\boldsymbol{H}}_{0}(f)=\sum_{k \in \mathbb{Z}} \boldsymbol{H}_{2 k} \mathrm{e}^{-\mathrm{i} 2 \pi f k}, \hat{\boldsymbol{H}}_{1}(f)=\sum_{k \in \mathbb{Z}} \boldsymbol{H}_{2 k+1} \mathrm{e}^{-\mathrm{i} 2 \pi f k},
\end{gathered}
$$

and the $2 n \times 2 n$ polyphase matrix

$$
\begin{aligned}
\boldsymbol{P}(f) & =\left[\begin{array}{ll}
\hat{\boldsymbol{G}}_{0}(f) & \hat{\boldsymbol{G}}_{1}(f) \\
\hat{\boldsymbol{H}}_{0}(f) & \hat{\boldsymbol{H}}_{1}(f)
\end{array}\right] \\
& =\left[\begin{array}{cc}
\hat{\boldsymbol{G}}\left(\frac{f}{2}\right) & \hat{\boldsymbol{G}}\left(\frac{f+1}{2}\right) \\
\hat{\boldsymbol{H}}\left(\frac{f}{2}\right) & \hat{\boldsymbol{H}}\left(\frac{f+1}{2}\right)
\end{array}\right] \cdot \frac{1}{2}\left[\begin{array}{cc}
1 & \mathrm{e}^{\mathrm{i} \pi f} \\
1 & -\mathrm{e}^{\mathrm{i} \pi f}
\end{array}\right] .
\end{aligned}
$$

As shown in $[33 \text {, Proposition } 1]^{1}$, the integer translates of the rows of $\boldsymbol{\Psi}(t)$ will form an orthonormal basis of $W_{0}$ if and only if $\left\{\boldsymbol{H}_{k}\right\}$ is chosen such that $\boldsymbol{P}(f)$ is paraunitary (i.e., $\left.\boldsymbol{P}(f) \boldsymbol{P}^{H}(f)=\boldsymbol{I}_{2 n}\right)$. Hence we are faced with the problem of completing a $2 n \times 2 n$ paraunitary matrix when given the first $n$ rows. Restricting ourselves to solutions corresponding to the minimum number of delays required for the filter's implementation, we may use the following approach.

First, we compute the decomposition [21, Thm. 9.2, Corr. 10.2]

$$
\left[\hat{\boldsymbol{G}}_{0}(f) \quad \hat{\boldsymbol{G}}_{1}(f)\right]=\boldsymbol{U}_{1} \boldsymbol{M}_{1}(f) \cdots \boldsymbol{M}_{d}(f) \mathrm{e}^{-\mathrm{i} 2 \pi \lambda f},
$$

where $\lambda \in \mathbb{Z}, d \leq L^{\prime} / 2$, and $\boldsymbol{U}_{1}=\left[\hat{\boldsymbol{G}}_{0}(0) \quad \hat{\boldsymbol{G}}_{1}(0)\right] \in \mathbb{R}^{n \times 2 n}$ satisfies $\boldsymbol{U}_{1} \boldsymbol{U}_{1}^{T}=\boldsymbol{I}_{n}$. An implementation of the above decomposition in Matlab is available as part of the mw toolbox [22]. The wavelet filters obtained will have length at most $L^{\prime}$.

We then complete the paraunitary matrix by taking

$$
\boldsymbol{P}(f)=\boldsymbol{U} \boldsymbol{M}_{1}(f) \cdots \boldsymbol{M}_{d}(f) \mathrm{e}^{-\mathrm{i} 2 \pi \lambda f},
$$

${ }^{1}$ Note that in [33] the scaling coefficients are scaled so that they sum to 2 rather than $\sqrt{2}$, and that the matrix product on the RHS is orthogonal iff the conditions of [33, Proposition 1] are satisfied. 

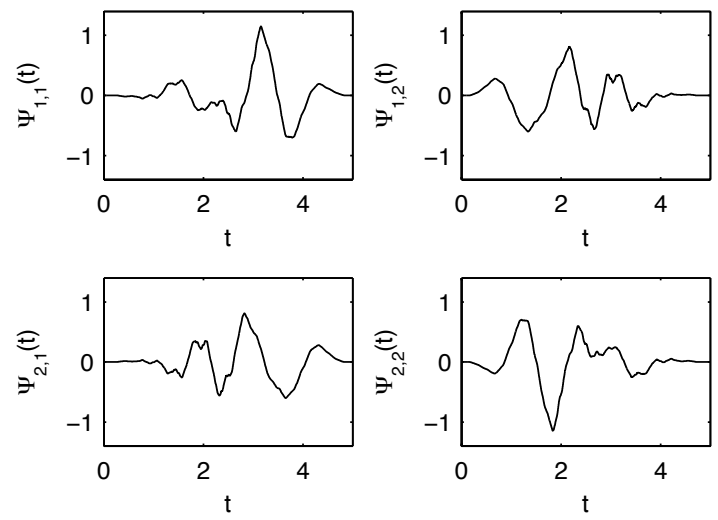

Fig. 2. Elements of $\boldsymbol{\Psi}(t)$ for the design with $L=6, A=3, n=2$ for the choice $x=\sqrt{5}$.

where $\boldsymbol{U}=\boldsymbol{P}(0)=\left[\begin{array}{l}\boldsymbol{U}_{1} \\ \boldsymbol{U}_{2}\end{array}\right] \in \mathbb{R}^{2 n \times 2 n}$ is unitary. If $\left\{\boldsymbol{G}_{k}\right\}$ is a MVSF , then it satisfies the scaling condition (5) and has one vanishing moment by Proposition 5 , hence from (19), $\hat{\boldsymbol{G}}_{0}(0)=$ $\hat{\boldsymbol{G}}_{1}(0)=\boldsymbol{I}_{n} / \sqrt{ } 2$, and $\boldsymbol{U}_{1}=\left[\begin{array}{ll}\boldsymbol{I}_{n} & \boldsymbol{I}_{n}\end{array}\right] / \sqrt{ } 2$. We may then choose $\boldsymbol{U}=\left[\begin{array}{cc}\boldsymbol{I}_{n} & \boldsymbol{I}_{n} \\ \boldsymbol{I}_{n} & -\boldsymbol{I}_{n}\end{array}\right] / \sqrt{ } 2$. (Note that if $\left\{\boldsymbol{H}_{k}\right\}$ is a valid wavelet filter, then so is $\left\{\boldsymbol{O H}_{k}\right\}$ for any orthogonal matrix $\boldsymbol{O}$.) For the $L=6, A=3, n=2$, scaling filter of (16) the corresponding wavelet filter coefficients for $x=\sqrt{5}$ are

$$
\begin{aligned}
& \boldsymbol{H}_{0}=\frac{1}{176 \sqrt{2}}\left[\begin{array}{cc}
-11+9 \sqrt{5} & 10 \sqrt{2}+11 \sqrt{10} \\
10 \sqrt{2}-11 \sqrt{10} & -11-9 \sqrt{5}
\end{array}\right], \\
& \boldsymbol{H}_{1}=\frac{1}{176 \sqrt{2}}\left[\begin{array}{cc}
55-27 \sqrt{5} & -30 \sqrt{2}-11 \sqrt{10} \\
-30 \sqrt{2}+11 \sqrt{10} & 55+27 \sqrt{5}
\end{array}\right], \\
& \boldsymbol{H}_{2}=\frac{1}{88 \sqrt{2}}\left[\begin{array}{cc}
-55+9 \sqrt{5} & 10 \sqrt{2}-11 \sqrt{10} \\
10 \sqrt{2}+11 \sqrt{10} & -55-9 \sqrt{5}
\end{array}\right],
\end{aligned}
$$

with $\boldsymbol{H}_{k}=\boldsymbol{O}_{0} \boldsymbol{H}_{5-k} \boldsymbol{O}_{0}$ for $k=3,4,5$, where we note the absence of a transpose on the right-most $\boldsymbol{O}_{0} . \boldsymbol{\Psi}(t)$ was computed from these $\boldsymbol{H}_{k}$ 's using the methodology in [30, Appendix A(b)] and is shown in Fig. 2.

A more general method which allows the construction of any valid FIR wavelet filter for a given scaling filter, is described in [34]. 


\section{QUATERNION WAVELETS}

In this paper, the terms "quaternion wavelet" and "quaternion scaling function" refer to functions in $L^{2}(\mathbb{R}, \mathbb{H})$ which we associate with an MRA of $L^{2}(\mathbb{R}, \mathbb{H})$. These should not be confused with similarly-named wavelets in the literature. For example, [7] defines quaternion wavelets whose components are 2D Hilbert transforms of real wavelets. These wavelets generate redundant frames of $L^{2}\left(\mathbb{R}^{2}, \mathbb{R}\right)$ and are used to analyse greyscale images. Such quaternion wavelets are equivalent to a group of complex wavelets up to a unitary linear transformation, as shown in [7, Section 5.2]. Another way in which such wavelets are related to complex wavelets is that the scaling function can be decomposed as as $\Phi(x, y)=\phi(x) \mathrm{e}^{\pi \mathrm{k} / 4} \phi(y) \mathrm{e}^{-\pi \mathrm{k} / 4}$ where $\phi$ is a complex scaling function (and similarly for the wavelet functions). Another example of quaternion wavelet admitting such a decomposition is given by the quaternion Gabor wavelets which are used in [6] for optical flow estimation of greyscale images.

\section{A. Matrix Representation}

When designing $2 \times 2$ scaling coefficients, we can impose the complex-structure conditions $g_{1,1, k}=g_{2,2, k}, g_{1,2, k}=-g_{2,1, k}$ to construct complex-structured MVWs. When applied to a signal whose values are complex-structured matrices, by isomorphism the resulting matrix wavelet transform is equivalent to a complex wavelet transform; see (17).

Quaternions $\mathbb{H}=\{a+b \mathrm{i}+c \mathrm{j}+d \mathrm{k}: a, b, c, d \in \mathbb{R}\}$, where $1, \mathrm{i}, \mathrm{j}, \mathrm{k}$ are the four basis elements, have a similar representation as quaternion-structured matrices in $\mathbb{R}^{4 \times 4}$, with the isomorphism

$$
a+b \mathrm{i}+c \mathrm{j}+d \mathrm{k} \mapsto\left[\begin{array}{cccc}
a & -b & -c & -d \\
b & a & -d & c \\
c & d & a & -b \\
d & -c & b & a
\end{array}\right] .
$$

By imposing the quaternion-structure conditions $g_{1,1, k}=g_{2,2, k}=g_{3,3, k}=g_{4,4, k}, g_{1,2, k}=g_{3,4, k}=$ $-g_{2,1, k}=-g_{4,3, k}, g_{1,3, k}=g_{4,2, k}=-g_{2,4, k}=-g_{3,1, k}, g_{1,4, k}=g_{2,3, k}=-g_{3,2, k}=-g_{4,1, k}$, we can design quaternion-structured MVWs and hence design quaternion wavelets.

Remark 3: Under the isomorphisms (17) and (20), complex/quaternion conjugation corresponds to matrix transposition.

A complex/quaternion-structured matrix is completely determined by its first row. Hence, when computing the matrix DWT of a complex/quaternion-structured matrix-valued sequence 
with a complex/quaternion-structured filter, it is sufficient to calculate only the first row at each iteration. If we do so, then the structured-matrix DWT requires the same number of (real) operations as a DWT implemented using complex/quaternion multiplication.

$\mathbb{H}$ is isomorphic to $\mathbb{R}^{1 \times 4}$ as a vector space. Hence, we may treat quaternion MRA (of $L^{2}(\mathbb{R}, \mathbb{H})$ ) as a special case of VMRA on $L^{2}\left(\mathbb{R}, \mathbb{R}^{1 \times 4}\right)$, with the additional condition that the $4 \times 4$ matrixvalued scaling function $\mathbf{\Phi}$ whose rows generate the VMRA must have quaternion structure (20).

\section{B. Literature Review}

Quaternion wavelets are investigated in [5] and [17] by using two different but equivalent representations of quaternions as structured matrices in $\mathbb{C}^{2 \times 2}$. Their frequency-domain approach makes use of quaternion Fourier transforms which are poorly suited to the task. We can express the Fourier transforms used in $\mathrm{He}$ and $\mathrm{Yu}$ [17] and in Bahri [5] directly in terms of the quaternion scaling function $\phi: \mathbb{R} \rightarrow \mathbb{H}$ and quaternion scaling sequence $\left\{g_{\ell}\right\}$.

Firstly, from $\mathrm{He}$ and $\mathrm{Yu}[17$, eqns. (1.7), (2.1), (2.2) and (3.1)], we find that

$$
\begin{aligned}
\hat{\phi}^{\mathrm{HY}}(f) & =\int_{-\infty}^{\infty} \mathrm{e}^{-\mathrm{i} 2 \pi f t} \phi(t) \mathrm{d} t \\
\hat{G}^{\mathrm{HY}}(f) & =\sum_{\ell \in \mathbb{Z}} g_{\ell} \mathrm{e}^{-\mathrm{i} 2 \pi f \ell} .
\end{aligned}
$$

Note that since $\phi(t), g_{\ell} \in \mathbb{H}$, and quaternion multiplication is not commutative, the placement of the complex exponential is important. It is claimed in [17] that

$$
\hat{\phi}^{\mathrm{HY}}(f)=\frac{1}{\sqrt{2}} \hat{G}^{\mathrm{HY}}\left(\frac{f}{2}\right) \hat{\phi}^{\mathrm{HY}}\left(\frac{f}{2}\right) .
$$

This does not hold if neither $\phi(t)$ nor the $g_{\ell}$ are complex because of the non-commutativity of quaternions. Consequently, the quaternion-valued wavelets in [17] are invalid. With the exception of the Haar filter, none of the scaling filters given are orthogonal to their even shifts. For example, the third design for $L=4$ is

$$
\begin{gathered}
g_{0}=0, g_{1}=\frac{1}{8 \sqrt{2}}(2-\sqrt{3} \mathrm{j}-3 \mathrm{k}), \\
g_{2}=\frac{1}{\sqrt{2}}, g_{3}=\frac{1}{8 \sqrt{2}}(6+\sqrt{3} \mathrm{j}+3 \mathrm{k}) .
\end{gathered}
$$

With $\bar{q}=a-b \mathrm{i}-c \mathrm{j}-d \mathrm{k}$ being the conjugate of $q \in \mathbb{H}$, for orthogonality we require the equivalent of (6), namely

$$
\sum_{k=0}^{L^{\prime}-1-2 m} g_{k} \bar{g}_{k+2 m}=\delta_{m, 0}, m=0, \ldots,\left(L^{\prime} / 2\right)-1
$$


with $L^{\prime}=L=4$. But, $g_{0} \bar{g}_{2}+g_{1} \bar{g}_{3}=g_{1} \bar{g}_{3}=-\frac{1}{16}(\sqrt{3} \mathrm{j}+3 \mathrm{k}) \neq 0$.

Secondly, for [5, eqns. (20) and (33)]

$$
\begin{gathered}
\hat{\phi}^{\text {Bahri }}(f)=\int_{-\infty}^{\infty} \phi(t) \mathrm{e}^{-\mathrm{k} 2 \pi f t} \mathrm{~d} t \\
\hat{G}^{\text {Bahri }}(f) \propto \sum_{\ell \in \mathbb{Z}} g_{\ell} \mathrm{e}^{-\mathrm{k} 2 \pi f \ell}
\end{gathered}
$$

respectively. [5, eqn. (34)] translates in our notation to

$$
\hat{\phi}^{\text {Bahri }}(f)=\hat{G}^{\text {Bahri }}\left(\frac{f}{2}\right) \hat{\phi}^{\text {Bahri }}\left(\frac{f}{2}\right) .
$$

However, the proof given in [5] requires commutativity between $\mathrm{e}^{-\mathrm{k} \pi f \ell}$ and $\phi(2 t-\ell)$, so again the given frequency-domain dilation equation does not hold in general.

In the structured-matrix approach to quaternion wavelets, we use the Fourier transform for matrix functions (3). The matrix Fourier transform can then be interpreted as a type of biquaternion Fourier transform, where we represent biquaternions as quaternion-structured $4 \times 4$ matrices with complex entries. (Biquaternions, which correspond to the Clifford algebra $\mathcal{C} l_{0,2}(\mathbb{C})$ are of the form $\{a+b \mathrm{i}+c \mathrm{j}+d \mathrm{k}: a, b, c, d \in \mathbb{C}\}$.) The Fourier theory for quaternion wavelets will be directly equivalent, by isomorphism to our matrix Fourier transform, whether we use the biquaternion Fourier transform, or choose to represent quaternions as structured matrices in $\mathbb{C}^{2 \times 2}$, or in $\mathbb{R}^{4 \times 4}$ and use the matrix Fourier transform (3).

Remark 4: For complex wavelets the usual $1 \times 1$ complex Fourier transform is not directly equivalent to the Fourier transform for complex structured $2 \times 2$ real matrices.

\section{Orthogonal Similarity}

For any quaternion $q=a+b \mathrm{i}+c \mathrm{j}+d \mathrm{k}$ there exists a unit quaternion $u$ such that

$$
u q \bar{u}=a+\left(b^{2}+c^{2}+d^{2}\right)^{1 / 2} \mathrm{i} .
$$

Under the real matrix representation (20), unit quaternions are orthogonal matrices so the 3D rotation $q \mapsto u q \bar{u}$ is an OST. Hence, similarly to how we used Lemma 1 in the general matrix case, we can assume up to orthogonal similarity that a quaternion-structured orthogonal MVSF has $\boldsymbol{G}_{0}$ block-diagonal (via (20), since $g_{3,1,0}=g_{4,1,0}=0$ because the coefficients of $\mathrm{j}$ and $\mathrm{k}$ are both zero in (23)). By then applying yet another OST of the form $q \mapsto u q \bar{u}$, with $u=\exp \left\{-\frac{1}{2} \tan \left(\frac{g_{3,1,1}}{g_{4,1,1}}\right) \mathrm{i}\right\}$, we can furthermore assume up to orthogonal similarity that $g_{3,1,1}=0$. 
Also note that real and complex filters are trivial quaternion filters, since they map to diagonal and block-diagonal matrices respectively under the isomorphism (20).

\section{Trivial Quaternion Wavelets}

Quaternion wavelets designed using the biquaternion Fourier transform are discussed in [25]. They give 3 designs of symmetric quaternion scaling filters by solving by hand the scaling, orthogonality and vanishing moments constraints on the coefficients. However, all designs in [25] are orthogonally similar to complex scaling filters and hence trivial.

The first design of [25], with $L=4, A=1$, gives the family of filters which are orthogonally similar to the symmetric complex scaling filters with coefficients

$$
g_{0}=g_{3}=x+y \mathrm{i} ; \quad g_{1}=g_{2}=(1 / \sqrt{2})-x-y \mathrm{i}
$$

where $y=\left[(x / \sqrt{2})-x^{2}\right]^{1 / 2}$ and $x \in[0,(1 / \sqrt{2})]$ is a free parameter. The second design, with $L=6$ and $A=2$ gives the family of filters which are orthogonally similar to the symmetric complex Daubechies filter of length 6. Hence the design actually satisfies $A=3$. The third design, with $L=8$ and $A=3$ is the family of filters which are orthogonally similar to the complex scaling filter

$$
\begin{aligned}
& g_{0}=g_{7}=[-155+\sqrt{1583470} \mathrm{i}] /[8448 \sqrt{2}] \\
& g_{1}=g_{6}=3 g_{0}+[1 /(16 \sqrt{2})] \\
& g_{2}=g_{5}=g_{0}+[5 /(16 \sqrt{2})] \\
& g_{3}=g_{4}=-5 g_{0}+[10 /(16 \sqrt{2})]
\end{aligned}
$$

Unfortunately, this filter does not satisfy the orthogonality constraints (22), since $g_{0} \bar{g}_{6}+g_{1} \bar{g}_{7}=$ $35 / 1056 \neq 0$. This is due to the fact that no symmetric quaternion scaling filter exists for $L=8$ and $A=3$, but [25] used only as many design equations as necessary to obtain a unique (up to OST) solution.

\section{A Non-trivial quaternion wavelet}

As a corollary of Propositions 8 and 9, there are no non-trivial quaternion wavelets with $L \leq 3$ and $A=1$ or $L=4$ and $A=2$. Using the computational method described earlier, we found that the only quaternion length 6 filters with 3 vanishing moments (respectively, length 8 and 4 vanishing moments) are the real and complex Daubechies filters of same length (up to orthogonal 
similarity). In practice, once a Gröbner basis is found for the equations describing a quaternion filter with $L=6$ and $A=3$, it can be checked that the polynomial $\sum_{k=0}^{L-1}\left(g_{3,1, k}^{2}+g_{4,1, k}^{2}\right)$ is equal to zero for all solutions (since this is equivalent to block-diagonal filters) and likewise for $L=8, A=4$. However, this is no longer true when $L=10$ and $A=5$, discussed next.

For $L=10$ and $A=5$ all non-trivial quaternion scaling filters are symmetric, and are given (up to orthogonal similarity) by

$$
\begin{aligned}
g_{0}=g_{9}= & C_{0}\left(y_{1}+y_{2} \mathrm{i}\right) \\
g_{1}=g_{8}= & C_{0}\left[\left(y_{1}-10\right)\right. \\
& \left.+y_{2}^{-1}\left(y_{2}^{2}+10 y_{1}-70\right) \mathrm{i}+y_{3} \mathrm{k}\right] \\
g_{2}=g_{7}= & C_{0}\left[\left(-4 y_{1}-14\right)\right. \\
& \left.-2 y_{2}^{-1}\left(2 y_{2}^{2}-15 y_{1}+105\right) \mathrm{i}+3 y_{3} \mathrm{k}\right] \\
g_{3}=g_{6}= & C_{0}\left[\left(-4 y_{1}+70\right)\right. \\
& \left.-2 y_{2}^{-1}\left(2 y_{2}^{2}-5 y_{1}+35\right) \mathrm{i}+y_{3} \mathrm{k}\right] \\
g_{4}=g_{5}= & C_{0}\left[\left(6 y_{1}+210\right)\right. \\
& \left.+2 y_{2}^{-1}\left(3 y_{2}^{2}-25 y_{1}+175\right) \mathrm{i}-5 y_{3} \mathrm{k}\right]
\end{aligned}
$$

where $C_{0}=1 /(256 \sqrt{ } 2)$ and $x$ is a real parameter and

$$
\begin{aligned}
& y_{1}=\sqrt{70} \cos (x) \\
& y_{2}=\sqrt{70} \sin (x) \\
& y_{3}=2 y_{2}^{-1}\left[60 y_{2}^{2}-8 y_{2}^{2} y_{1}+350 y_{1}-2975\right]^{1 / 2} .
\end{aligned}
$$

The range of $x$ is $1.0995 \lesssim x \lesssim 2.1764$, so that $y_{3}$ is real.

If we choose the two values of $x$ at the ends of its range, then $y_{3}=0$ and the resulting filters are the two different symmetric complex Daubechies filters of length 10.

If we choose $x=\pi / 2$, then $y_{3}=\sqrt{70}$ and

$$
\begin{aligned}
& g_{0}=g_{9}=C_{2} \mathrm{i} ; \quad g_{1}=g_{8}=-5 C_{1}+C_{2} \mathrm{k} \\
& g_{2}=g_{7}=-7 C_{1}-7 C_{2} \mathrm{i}+3 C_{2} \mathrm{k} \\
& g_{3}=g_{6}=35 C_{1}-5 C_{2} \mathrm{i}+C_{2} \mathrm{k} \\
& g_{4}=g_{5}=105 C_{1}+11 C_{2} \mathrm{i}-5 C_{2} \mathrm{k},
\end{aligned}
$$


where $C_{1}=\sqrt{2} / 256$ and $C_{2}=\sqrt{35} / 256$. The corresponding antisymmetric wavelet filter, computed using the method described in Section VII and the mw Matlab package [22], is given by

$$
\begin{aligned}
& h_{0}=-h_{9}=C_{3}[89 \sqrt{35} \mathrm{i}+35 \sqrt{2} \mathrm{j}-35 \sqrt{35} \mathrm{k}] \\
& h_{1}=-h_{8}=C_{3}[-480 \sqrt{2}+35 \sqrt{35} \mathrm{i}-175 \sqrt{2} \mathrm{j}+79 \sqrt{35} \mathrm{k}] \\
& h_{2}=-h_{7}=C_{4}[84 \sqrt{2}-91 \sqrt{35} \mathrm{i}+35 \sqrt{2} \mathrm{j}+\sqrt{35} \mathrm{k}] \\
& h_{3}=-h_{6}=C_{5}[35 \sqrt{2}+5 \sqrt{35} \mathrm{i}-\sqrt{35} \mathrm{k}] \\
& h_{4}=-h_{5}=C_{6}[-5040 \sqrt{2}+577 \sqrt{35} \mathrm{i}-245 \sqrt{2} \mathrm{j}+5 \sqrt{35} \mathrm{k}]
\end{aligned}
$$

where $C_{3}=1 / 24576, C_{4}=1 / 3072, C_{5}=1 / 256$, and $C_{6}=1 / 12288$. The resulting scaling and wavelet functions are depicted in Figure 3. Here the labelling of the plots reflects (20), e.g., the subscript 4, 1 in $\boldsymbol{\Phi}_{4,1}(t)$ refers to the 4th row and 1st column of the quaternion-structured matrix in $(20)$.

\section{Xi. A Characterization for Daubechies Matrix Wavelets}

In what follows we shall call $n \times n$ wavelets of even length $L^{\prime}=L$ with $L / 2$ vanishing moments by the name 'Daubechies Matrix Wavelets' (DMW) as the $L / 2$ vanishing moments property is shared by the seminal scalar wavelets design in [14]. For a DMW let $\boldsymbol{G}(z)$ be the $z$-transform of the scaling filter. Then we can write the orthonormality condition (6) as $\boldsymbol{G}(z) \boldsymbol{G}^{T}\left(z^{-1}\right)+$ $\boldsymbol{G}(-z) \boldsymbol{G}^{T}\left(-z^{-1}\right)=2 \boldsymbol{I}_{n}$ or as

$$
\boldsymbol{Q}(z)-\boldsymbol{Q}(-z)=z^{L-1} 2 \boldsymbol{I}_{n}
$$

where we define the polynomial matrix $\boldsymbol{Q}(z)$ as $\boldsymbol{Q}(z)=z^{L-1} \boldsymbol{G}(z) \boldsymbol{G}^{T}\left(z^{-1}\right)$. Equation (24) implies that either the polynomials in the off-diagonal entries of $\boldsymbol{Q}(z)$ are zero, or they contain only even powers of $z$. Consider the latter. A polynomial contains only even powers iff all roots appear in the form $r,-r$. From the vanishing moments condition, the off-diagonal polynomials of $\boldsymbol{G}(z) \boldsymbol{G}^{T}\left(z^{-1}\right)$ must have $L$ roots at -1 , which implies that they must have $L$ roots at 1 . However, the degree of these polynomials is at most $2 \times(L-1)=2 L-2$, which is a contradiction. Hence the off-diagonal entries of $\boldsymbol{Q}(z)$ must be zero, i.e., $\boldsymbol{Q}(z)$ is diagonal.

The diagonal entries of $\boldsymbol{Q}(z)$ satisfy the corresponding equations for the scalar Daubechies wavelet of length $L$. Hence $\boldsymbol{Q}(z)=q(z) \boldsymbol{I}_{n}$, where $q(z)=z^{L-1} g(z) g\left(z^{-1}\right)$ and $g(z)$ is the $z$ transform of a Daubechies scaling filter of length $L$. This implies that the rational polynomial 
matrix $\boldsymbol{U}(z)=\boldsymbol{G}(z) / g(z)$ is (normalized) paraunitary since $\boldsymbol{U}(z) \boldsymbol{U}^{T}\left(z^{-1}\right)=\boldsymbol{I}_{n}$.

Proposition 10: Every $n \times n$ Daubechies matrix wavelet $\left\{\boldsymbol{G}_{k}\right\}$ of length $L$ is of the form $\hat{\boldsymbol{G}}(f)=\hat{\boldsymbol{U}}(f) \hat{g}(f)$, where $\left\{g_{k}\right\}$ is the Daubechies wavelet of length $L$ and $\hat{\boldsymbol{U}}(f)$ is paraunitary $\left[29\right.$, p. 724] and satisfies $\hat{\boldsymbol{U}}(0)=\boldsymbol{I}_{n}$.

Remark 5: We may also obtain the wavelet filter $\hat{\boldsymbol{H}}(f)=\hat{\boldsymbol{U}}(f) \hat{h}(f)$, where $\left\{h_{k}\right\}$ is the scalar Daubechies wavelet filter of appropriate length. Applying a single level matrix-valued DMW transform to a signal is equivalent to applying a scalar Daubechies wavelet transform after prefiltering with the paraunitary transfer function $\hat{\boldsymbol{U}}(f)$. We note that this paraunitary prefiltering preserves energy at all frequencies $[29$, p. 725$]$. Also, when applied to white noise, paraunitary filtering outputs white noise with the same variance.

\section{Example: Attitude Quaternions}

When used to represent an orientation (rotation relative to a reference position), quaternions with a norm of unity are typically called orientation quaternions or attitude quaternions. Then

$$
q=a+b \mathrm{i}+c \mathrm{j}+d \mathrm{k}=\cos \frac{\theta}{2}+(x \mathrm{i}+y \mathrm{j}+z \mathrm{k}) \sin \frac{\theta}{2},
$$

where $\theta$ is the angle of rotation and $(x, y, z)$ the axis of rotation, and $x^{2}+y^{2}+z^{2}=1$.

In computer graphics, spherical linear interpolation (SLERP) [26] is a method for interpolating between unit quaternions which is commonly used to construct smooth animation curves. The top part of Fig. 4 gives the four components of a time series of attitude quaternions obtained by taking 65 random unit quaternions and using SLERP to join them smoothly.

Using both the scalar Daubechies wavelet of length 10 and our Daubechies (quaternion) construction from Fig. 3, we transformed the attitude quaternion time-series and applied hard vector thresholding to the wavelet coefficients. The bottom part of Fig. 4 compares the root mean squared error (RMSE) of the reconstructed signal in the scalar Daubechies and matrix (quaternion) Daubechies case. The results in Fig. 4 show that the quaternion wavelet significantly outperforms the scalar wavelet for a wide range of compression ratios. When $90 \%$ of the coefficients are zeroed out (compression ratio of 10:1), the RMSE is $27 \%$ lower for the quaternion wavelet $(1.06 \%$ of the signal's root energy is lost in the scalar wavelet reconstruction compared with $0.77 \%$ for the quaternion wavelet). When the percentage of coefficients set to zero is moderate, the scalar wavelets outperform the quaternion wavelets. 

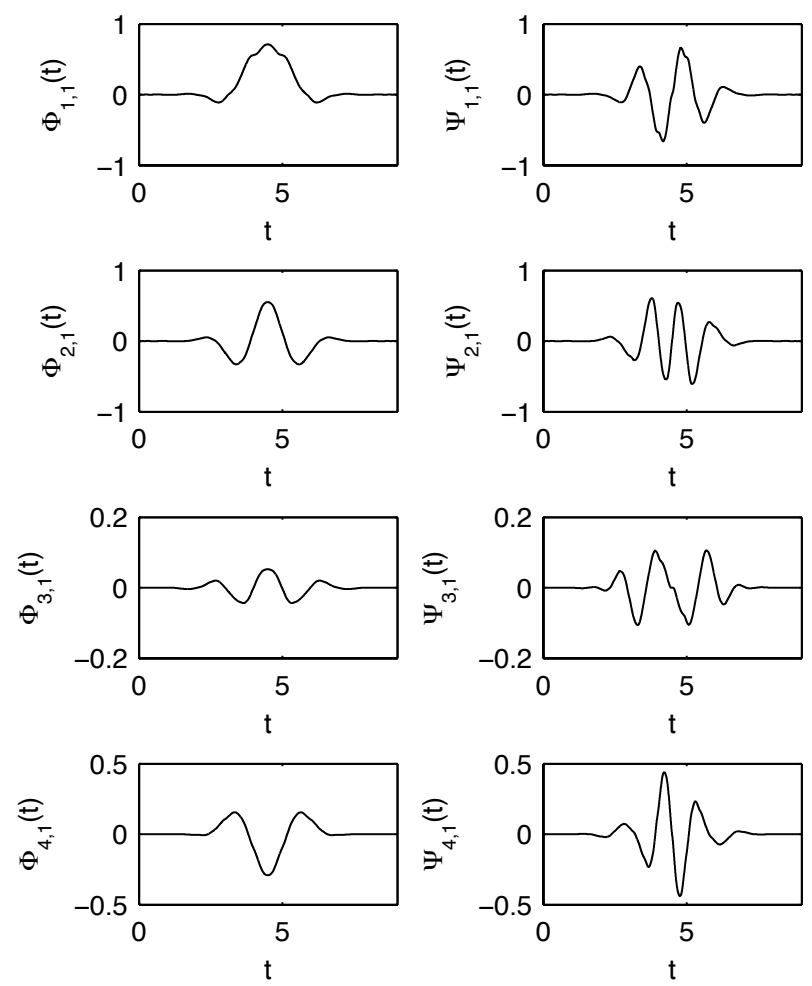

Fig. 3. Quaternion scaling and wavelet functions with $L=10, A=5$, and parameter $x=\pi / 2$.

\section{Closing Comments}

MVW transforms have some unique advantages - they can be applied to vector signals or vectorized scalar signals without the pre-processing usually required by multiwavelets, and have a theory that parallels the scalar case more closely than multiwavelets. However, there is a cost in terms of some achievable properties because of (5). For example, [28] describes a multiwavelet with $L=3$ and $A=2$, but such a construction would be impossible with MVWs by Corollary 2. In this sense MVWs are more similar to scalar/complex wavelets . Further, we can treat quaternion wavelets as a special case of matrix-valued wavelets.

Using a consistent approach, we have derived explicit non-trivial matrix-valued scaling and wavelet filters with three vanishing moments, and explicit non-trivial quaternion scaling and wavelet filters with five vanishing moments.

With a time series of attitude quaternions as an example, we have shown that it is possible for the non-trivial quaternion filter to concentrate more of a signal's energy in fewer coefficients, when compared with a scalar Daubechies filter of the same length. 

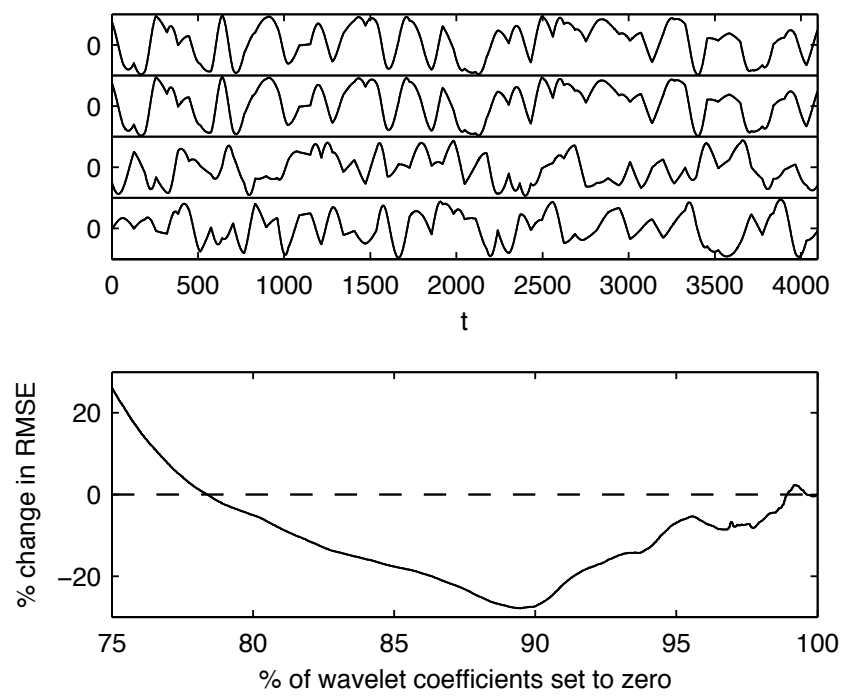

Fig. 4. Top: Synthetic time-series of attitude quaternions (real, i, j, and k components). Bottom: The percent change in RMSE when using our quaternion wavelet instead of the minimum phase scalar Daubechies wavelet of same length for wavelet thresholding.

Matrix-valued wavelets are a promising tool for the processing of vector-valued signals. However, more research is needed to understand when and how the additional degrees of freedom available in the design of MVWs can be effectively used to improve performance, and to predict for which signals and circumstances matrix-valued wavelets may offer a significant advantage over scalar wavelets.

The case of $2 \mathrm{D}$ signals such as images allows for yet more design freedom, and warrants a comparison of the type of quaternion wavelet presented here with the type used in [6], [7].

\section{APPENDiX}

\section{A. Proofs of Propositions}

We will denote the $i^{\text {th }}$ row of a matrix (or matrix-valued function) $\boldsymbol{F}$ by $\boldsymbol{F}^{(i, \bullet)}$, and for a set $V$, let $V^{(i, \bullet)}=\left\{\boldsymbol{F}^{(i, \bullet)}: \boldsymbol{F} \in V\right\}$.

\section{A.1 Proof of Proposition 1}

Let $\boldsymbol{F}^{(i, \bullet)} \in V^{(i, \bullet)}$. Let $\boldsymbol{M} \in \mathbb{R}^{n \times n}$ have $(j, i)$-entry equal to 1 and all other entries 0 . Then $\boldsymbol{F}^{(i, \bullet)}=(\boldsymbol{M} \boldsymbol{F})^{(j, \bullet)} \in V^{(j, \bullet)}$. Hence $V^{(i, \bullet)}=V^{(j, \bullet)}=S \forall i, j$ and $V \subseteq S^{n}$. Let $\boldsymbol{F} \in S^{n}$. For $i=1, \ldots, n$, choose $\boldsymbol{F}_{i} \in V$ such that $\boldsymbol{F}_{i}^{(i, \bullet)}=\boldsymbol{F}^{(i, \bullet)}$ and let $\boldsymbol{M}_{i} \in \mathbb{R}^{n \times n}$ have $(i, i)$-entry equal 
to 1 and all other entries 0. Then $\boldsymbol{F}=\sum_{i=1}^{n} \boldsymbol{M}_{i} \boldsymbol{F}_{i} \in V$, and so $S^{n} \subseteq V$. Hence $V=S^{n}$. Linearity of $S=V^{(1, \bullet)}$ follows directly from that of $V$. For the converse, note that for any $\boldsymbol{M} \in \mathbb{R}^{n \times n}$ and $\boldsymbol{F} \in S^{n},(\boldsymbol{M F})^{(i, \bullet)}$ is a linear combination of the rows of $\boldsymbol{F}$.

\section{A.2 Proof of Proposition 2}

For the "if" case, by Proposition 1 we can write $V_{j}=S_{j}^{n}$. For the "only if" case, set $V_{j}=S_{j}^{n}$ (the uniqueness of this construction then follows from the "if" case). We need to show that closedness and conditions 1 to 5 are satisfied by $S_{j}^{n}$ iff they are satisfied by $S_{j}$. For closedness and conditions 1,3 and 4 this is trivial. For condition 2, note that a sequence $\boldsymbol{F}_{k} \in \bigcup_{j \in \mathbb{Z}} S_{j}^{n}=\left(\bigcup_{j \in \mathbb{Z}} S_{j}\right)^{n}$ converges to $\boldsymbol{F}$ in $L^{2}$ iff for each $i$ the sequence $\boldsymbol{F}_{k}^{(i, \bullet)}$ converges to $\boldsymbol{F}^{(i, \bullet)}$. For condition 5: A $n \times n$ matrix-valued function $\mathbf{\Phi}(t)$ has orthonormal integer translates iff $\left\langle\boldsymbol{\Phi}^{(i, \bullet)}(t-k), \boldsymbol{\Phi}^{(j, \bullet)}(t-l)\right\rangle=\delta_{i, j} \delta_{k, l} \forall k, l \in \mathbb{Z} \forall i, j=1, \ldots, n . \forall \boldsymbol{F}(t) \in V_{0}$ there exists a sequence $\boldsymbol{A}_{k} \in \mathbb{R}^{n \times n}$ such that $\boldsymbol{F}(t)=\sum_{k \in \mathbb{Z}} \boldsymbol{A}_{k} \boldsymbol{\Phi}(t-k)$ iff for $i=1, \ldots, n, \forall \boldsymbol{F}^{(i, \bullet)} \in V_{0}^{(i, \bullet)}=S_{0}$ there exist $n$ sequences $a_{i, j, k} \in \mathbb{R}$ such that $\boldsymbol{F}^{(i, \bullet)}(t)=\sum_{k \in \mathbb{Z}} \sum_{j=1}^{n} a_{i, j, k} \boldsymbol{\Phi}^{(j, \bullet)}(t-k)$.

\section{A.3 Proof of Proposition 6}

$\left\{\boldsymbol{O} \boldsymbol{G}_{k} \boldsymbol{O}^{T}\right\}$ satisfies the necessary constraints (5) and (6) and the vanishing moments condition (7) iff $\boldsymbol{G}_{k}$ does. By (4), the scaling function obtained from $\left\{\boldsymbol{O G}_{k} \boldsymbol{O}^{T}\right\}$ is $\boldsymbol{O} \boldsymbol{\Phi}(t) \boldsymbol{O}^{T}$. Now $\left\langle\boldsymbol{O} \boldsymbol{\Phi}(t-k) \boldsymbol{O}^{T}, \boldsymbol{O} \boldsymbol{\Phi}(t-l) \boldsymbol{O}^{T}\right\rangle_{n \times n}=\boldsymbol{O}\langle\boldsymbol{\Phi}(t-k), \boldsymbol{\Phi}(t-l)\rangle_{n \times n} \boldsymbol{O}^{T}=\delta_{k, l} \boldsymbol{I}_{n}$. But $\boldsymbol{F}(t)=$ $\sum_{k \in \mathbb{Z}} \boldsymbol{A}_{k} \boldsymbol{\Phi}(t-k)$ if and only if

$$
\boldsymbol{F}(t) \boldsymbol{O}^{T}=\sum_{k \in \mathbb{Z}} \boldsymbol{A}_{k} \boldsymbol{O}^{T}\left(\boldsymbol{O} \boldsymbol{\Phi}(t-k) \boldsymbol{O}^{T}\right)=\sum_{k \in \mathbb{Z}} \boldsymbol{A}_{k} \boldsymbol{\Phi}(t-k) \boldsymbol{O}^{T}
$$

Hence the integer translates of $\boldsymbol{O} \boldsymbol{\Phi}(t) \boldsymbol{O}^{T}$ form an orthonormal basis of $V_{0} \boldsymbol{O}^{T}$.

\section{A.4 Proof of Proposition 7}

By time-shifting and choosing $L$, we may assume without loss of generality that $\boldsymbol{G}_{0} \neq \mathbf{0}_{n \times n}$. The only length 1 filter satisfying (5) is $\left\{\sqrt{2} \boldsymbol{I}_{n} \delta_{0, k}\right\}$, which is not orthogonal. Let $\left\{\boldsymbol{G}_{k}\right\}$ be an orthogonal scaling filter which is symmetric (resp. SA) and has odd length $L \geq 3$. Since, for odd $L$, we have $\boldsymbol{G}_{L}=\mathbf{0}_{n \times n}$, the last orthogonality condition in (6) gives us $\boldsymbol{G}_{0} \boldsymbol{G}_{L-1}^{T}=\mathbf{0}_{n \times n}$, which when combined with the assumed symmetry, gives us $\boldsymbol{G}_{0} \boldsymbol{G}_{0}^{T}=\mathbf{0}_{n \times n}\left(\right.$ resp. $\left.\boldsymbol{G}_{0} \boldsymbol{P}_{n} \boldsymbol{G}_{0}^{T} \boldsymbol{P}_{n}=\mathbf{0}_{n \times n}\right)$. By considering the diagonal terms, this implies that $\left\|\boldsymbol{G}_{0}^{(i, \bullet)}\right\|^{2}=0$ for all $i$. Hence $\boldsymbol{G}_{0}=\mathbf{0}_{n \times n}$, which contradicts our earlier assumption. 


\section{ACKNOWLEDGEMENTS}

Paul Ginzberg thanks the EPSRC (UK) for financial support. Helpful review comments by the referees and Associate Editor were much appreciated.

\section{REFERENCES}

[1] S. Agreste and A. Vocaturo, "Multichannel wavelet scheme for color image processing." In Applied and Industrial Mathematics in Italy III: Selected Contributions from the 9th SIMAI Conference, Rome, Italy 15-19 September 2008, eds. E. de Bernardis, R. Spigler \& V. Valente. World Scientific Publishing Co. Series on Advances in Mathematics for Applied Sciences, vol. 82, pp. 1-12, 2009.

[2] S. Agreste and A. Vocaturo, "A new class of full rank filters in the context of digital color image processing." In Proceedings of the 10th European Congress of ISS, (V. Capasso et al. Editors), The MIRIAM Project Series, ESCULAPIO Pub. Co., Bologna, Italy, pp. 1-6, 2009.

[3] S. Bacchelli, M. Cotronei \& T. Sauer, "Multifilters with and without prefilters," BIT Numerical Mathematics, vol. 42, pp. 231-261, 2002.

[4] S. Bacchelli, M. Cotronei \& T. Sauer, "Wavelets for multichannel signals," Adv. in Appl. Maths., vol. 29, pp. 581-598, 2002.

[5] M. Bahri, "Construction of quaternion-valued wavelets," Matematika, vol. 26, pp. 107-114, 2010.

[6] E. Bayro-Corrochano, "The theory and use of the quaternion wavelet transform," J. Math. Imaging E Vision, vol. 24 , pp. 19-35, 2006.

[7] W. L. Chan, H. Choi and R. Baraniuk, "Quaternion wavelets for image analysis and processing." In Proceedings of the 2004 International Conference on Image Processing (ICIP-2004), Singapore, vol. 5, pp. 3057-3060, 2004.

[8] Q. J. Chen, Z. X. Cheng \& C. L. Wang, "Existence and construction of compactly supported biorthogonal multiple vector-valued wavelets," J. Appl. Math. and Comput., vol. 22, pp. 101-115, 2006.

[9] Q. Chen and Z. Shi, "Construction and properties of orthogonal matrix-valued wavelets and wavelet packets," Chaos, Solitons 6 Fractals, vol. 37, pp. 75-86, 2008.

[10] C. K. Chui and J. Lian, "A study of orthonormal multi-wavelets," Appl. Numer. Math., vol. 20, pp. 273-298, 1996.

[11] C. Conti, M. Cotronei \& T. Sauer, "Full rank positive matrix symbols: interpolation and orthogonality," BIT Numerical Mathematics, vol. 48, pp. 5-27, 2008.

[12] L. Cui, B. Zhai \& T. Zhang, "Existence and design of biorthogonal matrix-valued wavelets," Nonlinear Analysis: Real World Applications, vol. 10, pp. 2679-2687, 2009.

[13] L. Cui and T. Zhang, "m-band orthogonal vector-valued multiwavelets," J. Appl. Maths. and Comput., vol. 28, pp. 165-184, 2008.

[14] I. Daubechies, "Orthonormal bases of compactly supported wavelets," Comm. Pure and Applied Math., vol. 41, pp. 909-996, 1988.

[15] I. Daubechies, Ten Lectures on Wavelets. Philadelphia: SIAM, 1992.

[16] J. E. Fowler and L. Hua, "Wavelet transforms for vector fields using omnidirectionally balanced multiwavelets," IEEE Trans. Signal Process., vol. 50, pp. 3018-3027, 2002. 
[17] J. X. He and B. Yu, "Wavelet analysis of quaternion-valued time-series," Int. J. of Wavelets, Multiresolution and Inform. Process., vol. 3, pp. 233-246, 2005.

[18] C. Heil and D. Colella, "Matrix refinement equations: existence and uniqueness," The J. of Fourier Analy. and Appl., vol. 2, pp. 363-377, 1996.

[19] L. Hua and J. E. Fowler, "Technical details on a family of omnidirectionally balanced symmetric-antisymmetric multiwavelets." Technical report MSSU-COE-ERC-02-08, Engineering Research Center, Mississippi State University. citeseerx.ist.psu.edu/viewdoc/summary?doi=10.1.1.11.3802, 2002.

[20] L. Hua and J. E. Fowler, "Wavelet-based coding of time-varying vector fields of ocean-surface winds," IEEE Trans. Geosci. Remote Sensing, vol. 42, pp. 1283-1290, 2004.

[21] F. Keinert, Wavelets and Multiwavelets. Chapman and Hall/CRC, 2003.

[22] F. Keinert, http://orion.math.iastate.edu/keinert/book.html, 2004.

[23] J. Lebrun and I. Selesnick, "Gröbner bases and wavelet design," J. of Symbolic Comput., vol. 37, pp. 227-259, 2004.

[24] J.-M. Lina and M. Mayrand, "Complex Daubechies wavelets," Appl. and Comput. Harmonic Analysis, vol. 2, pp. 219-229, 1995.

[25] L. Peng and J. Zhao, "Quaternion-valued smooth orthogonal wavelets with short support and symmetry," in Advances in analysis and geometry: new developments using Clifford algebras, (T. Qian et al. Editors), Birkhäuser, pp. 365-376, 2004.

[26] K. Shoemake, "Animating rotation with quaternion curves," ACM SIGGRAPH, vol. 19, pp. 245-254, 1985.

[27] G. Strang and T. Nguyen, Wavelets and Filter Banks. Wellesley MA: Wellesley Cambridge Press, 1996.

[28] G. Strang and V. Strela, "Orthogonal multiwavelets with vanishing moments," J. Optical Eng., vol. 33, pp. 2104-2107, 1994.

[29] P. P. Vaidyanathan, Multirate Systems and Filter Banks. Englewood Cliffs NJ: Prentice Hall, 1993.

[30] A. T. Walden and A. Serroukh, "Wavelet analysis of matrix-valued time series," Proc. R. Soc. Lond. A., vol. 458, pp. 157-179, 2002.

[31] M. A. Westenberg and E. Thomas, "Denoising 2-D vector fields by vector wavelet thresholding," J. WSCG vol. 13 , pp. 33-40, 2005.

[32] X.-G. Xia, "Orthonormal matrix valued wavelets and matrix Karhunen-Loève expansion." In Contemporary mathematics volume 216: wavelets, multiwavelets, and their applications (eds A. Aldroubi \& E. B.Lin), pp. 15975, Providence, RI: American Mathematical Society, 1997.

[33] X.-G. Xia and B. W. Suter, "Vector-valued wavelets and vector filter banks," IEEE Trans. Signal Process., vol. 44, pp. 508-18, 1996.

[34] X.-G. Xia and B. W. Suter, "FIR paraunitary filter banks given several analysis filters: factorizations and constructions," IEEE Trans. Signal Process., vol. 44, pp. 720-723, 1996.

[35] B. M. Yu, "On existence of matrix-valued wavelets," Advanced Materials Research, vol. 282-283, pp. 153-156, 2011. 


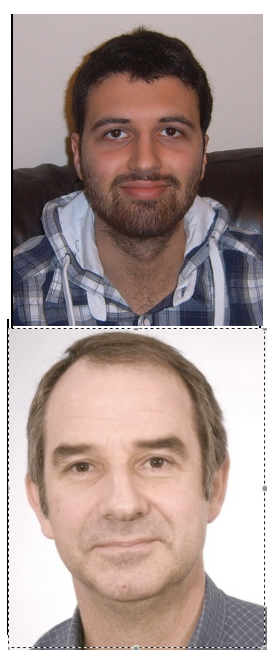

Paul Ginzberg received the M.Sci. degree in mathematics in 2009 from Imperial College London, U.K., where he is currently pursuing a Ph.D. in statistics. His interests include quaternion signal processing and wavelets.

Andrew T. Walden (A'86-M'07-SM'11) received the B.Sc. degree in mathematics from the University of Wales, Bangor, U.K., in 1977, and the M.Sc. and Ph.D. degrees in statistics from the University of Southampton, Southampton, U.K., in 1979 and 1982, respectively. He was a Research Scientist at BP, London, U.K., from 1981 to 1990, and then joined the Department of Mathematics at Imperial College London, London, U.K., where he is currently a Professor of statistics. 